\title{
EVALUACIÓN DE NECESIDADES PARA LA INTERVENCIÓN ORIENTADORA EN EL DESARROLLO PROFESIONAL FEMENINO: LA INSERCIÓN SOCIO-LABORAL DE MUJERES JÓVENES
}

\author{
NEEDS ASSESSMENT FOR WOMEN CAREER DEVELOPMENT: \\ FACILITATING YOUNG WOMEN SOCIAL AND WORK TRANSITION
}

\author{
Magdalena Suárez, Ortega* \\ Universidad de Sevilla
}

\begin{abstract}
RESUMEN
El acceso al empleo y el mantenimiento del puesto de trabajo son temas de gran relevancia social. Tanto jóvenes que eligen o demandan su primer empleo, personas que abandonaron sus puestos y quieren reinsertarse en el mundo laboral, como las que necesitan de una re-cualificación para el mantenimiento del puesto, encuentran serias dificultades en el proceso de inserción y desarrollo profesional. No obstante, existen determinados sectores de población, como el femenino, que encuentran especiales barreras que obstaculizan dichos procesos.

En el trabajo que sigue planteamos la necesidad de integrar una perspectiva de género en la orientación profesional, donde se tengan en cuenta los diferentes condicionantes que las mujeres encuentran a la hora del acceso al empleo y del mantenimiento del puesto. A partir de él, detectamos algunas de las necesidades que posee un colectivo de mujeres jóvenes para su inserción sociolaboral, que limitan de manera clara sus procesos de desarrollo personal-profesional.
\end{abstract}

Palabras clave: Evaluación de necesidades, Orientación Profesional, Perspectiva de Género.

\begin{abstract}
Accessing the labour market and maintaining a job are two important priorities that concern today's society. Serious difficulties are faced by young people searching and applying for their first job, and by those returning to work. Also, those already in a job can be affected when they must re-train in order to mantein their position. These problems tend to arise in the employee selection process and
\end{abstract}

* Es Becaria de Investigación de la Universidad de Sevilla. Ha participado en varias investigaciones sobre el sexismo en el ámbito educativo y sobre la validación de instrumentos diagnósticos. Actualmente su trabajo se centra en el desarrollo profesional femenino. 
in professional development. However, there are certain members of society who fall into particular social categories, such women, for which the limitations are greater. This study highlights the importance of a better understanding of the gender factor in the career guidance for women. We present training and guidance needs assessment for a group of young women who were looking to access to job.

Key Words: Needs Assessment, Career Guidance, Gender Perspective.

\section{El Desarrollo Profesional Femenino: por qué y para qué trabajar desde una Perspectiva de Género}

La división de espacios sociales ha sido tradición en nuestras vidas marcando el desarrollo personal-profesional tanto de hombres como de mujeres. Este aspecto ha tenido y tiene importantes repercusiones de cara a la formación e inserción en el mercado laboral y productivo en igualdad de oportunidades (Chait y Shibley, 2001). Tradicionalmente, el hombre es el que se ha encargado del sustento económico de la familia, mientras que la mujer se encargaba más de la realización de tareas reproductivas.

Esta división sexual del trabajo tiene importantes repercusiones de cara a la inserción laboral femenina (Poal, 1993). De manera tal que, aunque el acceso al empleo sea de por sí un proceso que reviste dificultades tanto para hombres como para mujeres, éstas se encuentran con una serie de barreras y /o limitaciones "añadidas" a dicho proceso y que obstaculizan su desarrollo profesional (Padilla, 2001; 2002 ${ }^{1}$ ). Por este motivo, las mujeres son consideradas un colectivo prioritario para participar en acciones formativas con el objeto de potenciar y facilitar su acceso al empleo $^{2}$ y de crear cauces para el mantenimiento de los mismos, fomentando puestos de trabajo estables ${ }^{3}$.

Debido a las limitaciones con las que se encuentran para acceder y mantener un puesto de trabajo, las mujeres son las primeras en abandonarlos cuando en sus vidas personales-familiares aparecen otras variables, como el matrimonio o la convivencia con la pareja, o el nacimiento de los hijos (Marí-Klose y Nos Colom, 1999). No queremos decir con ello que dichas variables sean causa-efecto pero, de acuerdo con estas autoras, sí las consideramos factores condicionantes de tales procesos.

A todas estas limitaciones tenemos que añadir las muchas desigualdades que se producen en materia laboral (Rodríguez-Piñero y Castellano, 2003). Por poner algunos ejemplos, los contratos que se realizan al colectivo femenino son más escasos y la discriminación salarial es una constante en la sociedad actual; "las mujeres ganan menos que los hombres, tanto en trabajos manuales como en los no manuales, con diferencias de entre un $15 \%$ y un 40\% respectivamente" (Fondo Social Europeo, 1997, cit. por Calvet, 2000: 22).

\footnotetext{
${ }^{1}$ Padilla, M.T. (2002). Desarrollo profesional femenino: la identidad de la mujer en el mundo laboral. En E. Moreno y S. Villegas (Eds.), Introducción a los estudios de la mujer. Una mirada desde las ciencias sociales (pp. 73-95). Huelva: Diputación Provincial / Seminario de Estudios de la Mujer de la Universidad de Huelva.

2 Real Decreto 631/1993, de 3 de mayo (B.O.E. 4-05-1993), por el que se regula el Plan Nacional de Formación e Inserción Profesional (aprobado por el Real Decreto 1618/1990 de 14 de diciembre), en cuyo objeto (art.1) se considera a las mujeres como colectivo prioritario y preferente en sus actuaciones.

${ }^{3}$ Orden de 16 de septiembre de 1998 (B.O.E. 29-09-1998) para el fomento de empleo estable de mujeres en las profesiones y ocupaciones con menor índice de empleo femenino.
} 
Los salarios femeninos son más bajos aunque se realice el mismo trabajo. Además, muchas mujeres acceden a los trabajos de manera temporal y por necesidad, entendiendo su rol laboral como complemento al sueldo del marido. De esta forma,

"la lógica de la división sexual del trabajo, recreada en un espacio que remueva el reparto de funciones y jerarquías, convierte a las mujeres en adultos dependientes de los adultos proveedores de renta. Sabemos que ante una oportunidad de promoción, un trabajo interesante pero encorsetado en una jornada prolongada, la mujer ha de optar ${ }^{4}$ entre dos realidades. En una de ellas no goza del beneficio de la dispensa, o se administra una sustitución, la paga, o simplemente renuncia. "Ganar menos" es femenino" (Murillo, 1998:83)5.

Por otro lado, la presencia de las mujeres en los puestos de dirección es mucho menor, accediendo a determinados empleos y no a otros (Carrasco, 2002). Así, la tipificación de profesiones y la segregación sectorial y ocupacional son claras. Al respecto nos hacemos una serie de interrogantes: ¿cuántas mujeres están, "o se consideran”, trabajadoras u ocupadas?, ¿qué tipos de trabajos realizan y en qué se ocupan?, ¿en qué condiciones lo desempeñan y a costa de qué? ...

Las mujeres que realizan un trabajo remunerado, las que se consideran trabajadoras "activas", realizan trabajos claramente diferentes a los de los hombres, concentrándose en ciertas ramas de actividad y en ciertos empleos, normalmente de menor cualificación y responsabilidad (Instituto Andaluz de la Mujer, 2001; Instituto de Estadística de Andalucía, 2002). De esta manera, el perfil del trabajador es de una persona sin ningún tipo de cargos ni responsabilidades familiares, autónoma, varón y sin "riesgo" de maternidad.

Como podemos apreciar, la vida laboral de las mujeres supone un modelo bien distinto al de los hombres por varias cuestiones, entre las que se destaca el trabajo familiar (relaciones trabajo-familia) (Cigarini, 2000). Muchas mujeres que trabajan fuera de este ámbito están empleadas a doble jornada, trabajando tanto fuera como dentro del hogar. Este aspecto, en nuestra opinión, es fundamental destacarlo ya que se sigue considerando el ámbito familiar como deber principal de las mujeres, a lo que se unen las cargas familiares.

Esta atribución es así considerada no sólo por buena parte del colectivo masculino. En muchas ocasiones, son las propias mujeres las que, de manera consciente o inconsciente, se atribuyen y acaparan estas responsabilidades. Lejos de ser comunes para todos y todas, las hacen obligaciones y deberes únicamente de su sexo. En todo ello, como aportan Lent y Worthington (2000), los patrones culturales se convierten son referentes esenciales que intervienen en el desarrollo profesional femenino.

\footnotetext{
${ }^{4}$ La cursiva es nuestra. La mujer no opta entre dos realidades, sino que se queda con las dos. Esta toma de decisiones (el acceso y el mantenimiento del puesto de trabajo) es compleja ya que existen mecanismos ocultos que hacen que las mujeres no separen los ámbitos privado-público o el trabajo-familia de la misma manera que lo hace el hombre. Además, su compromiso, auto-obligación, sentimiento de necesidad y de culpabilidad si no realiza determinadas tareas, o no dedica tiempo a otras personas (padres /madres, abuelos /as, hijos /as...) suele ser mayor que el que siente el colectivo masculino. Al respecto, deberíamos plantearnos cuáles son los mecanismos que hace que el trabajo de las mujeres no se desligue de las esferas del hogar ni de otras ocupaciones / funciones tradicionalmente desempeñadas. De otro lado, consideramos prioritario cuestionarnos los factores que dificultan una real y efectiva conciliación de la vida personal-familiar y profesional de mujeres y de hombres.

${ }^{5}$ Murillo de la Vega, S. (1998). Nuevas profesiones y empleo femenino: un criterio de calidad. En Instituto Andaluz de la Mujer. Primeras jornadas Now de Andalucía /Segundo encuentro empleo Now de Andalucía, nuevas oportunidades para las mujeres. Instituto Andaluz de la Mujer.
} 
En este sentido, y desde las relaciones que se establecen entre las mujeres y el mercado de trabajo, ya sea remunerado o no, consideramos de especial relevancia prestar atención a las auto-percepciones que éstas tienen del rol de trabajadoras remuneradas y del de amas de casa y de madres. El primero, desde un significado de dependencia y complementariedad. El segundo, manifestado como gratuito, no reconocido, y realizado en función exclusiva de su sexo. Nos referimos al elemento subjetivo que el trabajo comporta, aspecto que posee repercusiones desde las diferencias de género apuntadas.

Por tanto, desde esta perspectiva de subjetividad femenina, es fundamental evaluar las necesidades con las que se encuentran las mujeres para su acceso al trabajo remunerado, así como regular las tareas dentro del trabajo familiar. Éstas tienen un peso decisivo en la toma de decisiones vitales/profesionales de las mujeres. Tener en cuenta estos condicionantes en nuestras actuaciones e integrar una Perspectiva de Género en el marco de la Orientación y de la Formación Profesional es un aspecto que consideramos clave de cara a cambios positivos, mejoras y avances en el desarrollo profesional femenino (LIKaDI Formación y Empleo. Instituto Andaluz de la Mujer, 2001).

\section{Diseño de la Investigación}

La investigación que realizamos precede al diseño de una intervención orientadora. Por tanto, se lleva a cabo con dicha intención, desarrollándose en momentos iniciales y previos a la misma. Consideramos el análisis de necesidades un primer paso fundamental antes de intervenir en cualquier contexto, o con cualquier colectivo, ya que aunque podamos tener una primera referencia ninguna determinación de necesidades es definitiva y completa. Éstas pueden variar en cualquier momento o aparecer otras nuevas que antes no existían (Kaufman, 1997).

El modelo de evaluación de necesidades en el que nos basamos es el modelo A.N.I.S.E. (Análisis de Necesidades de Intervención Socioeducativa) (Pérez-Campanero, 1991), el cual se compone de tres fases: conocimiento, diagnóstico y toma de decisiones, que se desarrollan a lo largo de once etapas, y cuyo objetivo es el estudio, en profundidad y desde diferentes ópticas, de una determinada problemática.

\section{Propósito y objetivos del estudio}

El propósito de nuestra investigación es, por tanto, evaluar las necesidades con las que un colectivo de mujeres jóvenes se encuentra a la hora de insertarse socio-laboralmente en su entorno. Todo ello de cara al diseño de una intervención psicopedagógica con dicho colectivo. De esta manera, los objetivos que nos planteamos son:

- Detectar necesidades reales, sentidas y expresadas por las destinatarias de la intervención con respecto a su presente-futuro profesional.

- Detectar necesidades normativas, definidas por las distintas profesionales que intervienen en el programa (orientadora sociolaboral, educadora y monitora), con respecto a las destinatarias y a su desarrollo profesional. 
- Conocer si se dan discrepancias entre las necesidades expresadas por las destinatarias $\mathrm{y}$ las definidas por las profesionales.

\section{Población y muestra}

La intervención desarrollada se ubica en el Centro Municipal de Información a la Mujer de San Juan de Aznalfarache (Delegación de la Mujer del Ayuntamiento de dicha localidad), concretamente, en el Departamento de Empleo y Formación de dicho centro. Éste lleva a cabo el Plan de Barriada (Santa Isabel) para la mejora de la misma y el aumento de su calidad de vida (prevención de situaciones de marginalidad, oferta formativa alternativa y de inserción laboral).

Teniendo en cuenta las necesidades concretas de la zona y las particularidades de las familias que viven en ella, se llevó a cabo una selección de las participantes en el programa por el Centro Municipal de Información a la Mujer. La selección de las alumnas no se realizó al azar, sino atendiendo a una serie de requisitos que se extrajeron de las necesidades del propio contexto: mujeres sin escolarizar, bajo nivel de formación (preferentemente que hubiesen abandonado los estudios) y su situación sociofamiliar, especialmente si estaban a cargo de familias monomarentales.

Por tanto, la población en la que intervenimos fue la Barriada Santa Isabel de San Juan de Aznalfarache. De aquí, y en función de la información aportada, se extrajo una muestra de veinte mujeres, que fueron las destinatarias de la intervención orientadora. Las edades de las mismas están comprendidas entre los 16 y los 22 años, correspondiendo estos a los valores mínimo y máximo del conjunto, respectivamente. La edad media de las chicas encuestadas es de 18,40 y la desviación típica igual a 1,818.

\section{Técnicas e Instrumentos para la Recogida de Información}

Se ha hecho uso tanto de una metodología cualitativa como cuantitativa, en función de los momentos y de las características del proceso evaluador. De esta manera, se complementan datos cualitativos y cuantitativos, lo que confiere al análisis y a la interpretación de los mismos mayor riqueza y profundidad. Para dar respuesta a los objetivos planteados en este trabajo, los procedimientos e instrumentos de recogida de información que utilizamos son diversos. Así, nos centramos en las técnicas de encuesta (cuestionario y entrevista), en el análisis de indicadores sociales y fuentes documentales y en la escala de conducta exploratoria ESCE (Rodríguez Moreno, 1999).

El "Cuestionario para la Detección de Necesidades Formativas de las Chicas" es un instrumento que diseñamos adaptado a los objetivos del estudio. Éste consta de doce preguntas en total, tanto cerradas como abiertas, en relación a la Orientación Profesional e Inserción Laboral. La finalidad de dicho cuestionario es la recogida de información complementaria desde la perspectiva de las chicas para detectar necesidades o carencias formativas sentidas para el acceso al mercado laboral.

Las entrevistas se realizan a diferentes profesionales (orientadora sociolaboral, educadora y monitora) que trabajaban con este grupo. Son entrevistas abiertas o en profundidad, cuyo objetivo básico es la obtención de información productiva desde estos agentes sobre la 
problemática objeto de estudio, en relación al grupo diana, y al contexto en que éste se inserta. Para el análisis de indicadores sociales y fuentes documentales tuvimos en cuenta un estudio -ya realizado- del contexto de la barriada, así como de la evaluación de un programa previo desarrollado en la misma (documentos internos del centro). Todos los datos recabados por estos medios nos fueron útiles para complementar y triangular la información obtenida mediante otros procedimientos.

Por último, en la escala utilizada (ESCE) se abordan temas relacionados con la exploración del mundo del trabajo (análisis de la conducta exploratoria). Es un instrumento compuesto por nueve dimensiones y trece sub-escalas. A este instrumento se responde de 1 a 5 según se esté muy poco de acuerdo o muy de acuerdo con los distintos enunciados de los que se compone. Los resultados del análisis y de la validación de la escala indican la fiabilidad de sus puntuaciones. Esto queda demostrado por el valor obtenido de toda la escala tras el cálculo del Alpha de Cronbach $(0,8967)$, que es uno de los indicadores más potentes para medir la consistencia interna. El análisis de consistencia por dimensiones nos aporta también índices de fiabilidad, que se sitúan entre 0,7336 y 0,8841 (Donoso, Figuera y Torrado, 2000).

\section{Análisis de la información}

El análisis de datos cuantitativos se realizó mediante el paquete estadístico SPSS. Por las características de nuestro estudio (muestra muy pequeña y variables con niveles de medida nominal y ordinal) se calcularon porcentajes y medianas para las variables procedentes del cuestionario y de la escala (ESCE), respectivamente. En cuanto al proceso de análisis de los datos cualitativos que seguimos, estos se analizaron por los procedimientos habituales: recogida de datos, transcripción, reducción de los mismos (procedimiento de saturación) e identificación, y posterior codificación e interpretación de las categorías extraídas (extracción y verificación de conclusiones).

\section{Resultados de la evaluación de necesidades}

\section{Necesidades extraídas del análisis del contexto de intervención}

Para evaluar las necesidades, como ya indicamos, nos servimos del análisis de indicadores sociales y fuentes documentales. Partimos de la importancia de tener un conocimiento profundo del contexto y de comprender dónde se ubican las cosas. Es decir, el entorno micro-social donde se desarrolla la intervención, ya que cada comunidad posee unos parámetros culturales específicos a partir de los cuales crece y evoluciona. Por tanto, este primer diagnóstico lo consideramos prioritario para detectar dónde se encuentran los problemas, no sólo físicamente, sino también según la mentalidad, el entorno, y la situación de las personas.

De dicho análisis genera una gran cantidad de datos. Sin embargo, en este trabajo nos limitamos a destacar algunos de los hallazgos, en nuestra opinión, más relevantes, y que nos son de utilidad de cara al diseño de la intervención orientadora desarrollada: 
- La localidad en cuestión presenta un hábitat disperso, asentándose en una serie de núcleos urbanos bien diferenciados geográfica, social, económica y culturalmente. Entre ellos existen diferencias importantes, tanto en lo que respecta a la estructura de las viviendas, como en cuanto al número y a las características de la población.

- Este aspecto parece que guarda cierta relación con el crecimiento de la localidad y con las diferencias a nivel social, económico y cultural de las personas que aquí viven.

- En este sentido, se observan núcleos urbanos con problemáticas específicas de paro, pobreza e inclusión social. En ellas se hace patente la necesidad de intervenir para la mejora y el desarrollo de las mismas, así como para la integración de esta población en el contexto social más amplio de la localidad.

- Nos situamos en un "pueblo dormitorio" cercano a la capital, caracterizado por la escasez de industrias. Sobresalen los pequeños comercios, lo que nos induce a pensar que la oferta de trabajo se queda en la propia familia y se cierran así las posibilidades de empleo a personas ajenas a dichos núcleos familiares.

- Las demandas sociales planteadas a diferentes niveles (educativo, social, económico) parecen mantener cierta conexión con el desarrollo de medios y de recursos a nivel local, con el equipamiento de ésta, así como con la situación de los mismos en puntos estratégicos. Nos referimos, por ejemplo, a la ubicación de Servicios Sociales y del Centro Municipal de Información a la Mujer próximos a la barriada en la que realizamos el estudio, u otros núcleos.

- La población oscila a 20.709 habitantes $^{6}$, de entre los cuales 10.716 son mujeres y 9.993 hombres. Observamos que el número de mujeres con el que cuenta la población sanjuanera es ligeramente superior al de hombres, aunque dicho incremento, si nos detenemos en la pirámide de población, se hace más notable a edades más avanzadas.

- La localidad de San Juan también cuenta con una población joven relevante, situándose la mayor parte de la misma en el intervalo de edad comprendido entre los 15 y los 35 años. El intervalo de edad que se presenta con mayor número de sujetos es el que comprende de los 25 a los 29 años, con 2.059 sujetos en total, de los que 999 son mujeres y 1.060 hombres. No obstante, en esta población joven se observa un descenso en los últimos quince años.

- Por características propias de la misma, muy poco término municipal, caracterizándose sus nuevas construcciones por las grandes aglomeraciones urbanas y la falta de espacios verdes y de recreo, la gente joven conforma sus núcleos de residencia en otras localidades cercanas a la capital.

- Por otro lado, según datos ofrecidos por el INEM (febrero de 2001), el número de demandantes activos de empleo es de 2.554 en total, de entre los cuales 1.308 son mujeres y 1.246 son hombres. El tramo de edad en que la demanda de empleo se hace más notable es el que va de los 20 a los 24 años, tanto para hombres como para mujeres, aunque estas últimas superan ligeramente a los primeros en este aspecto. Se observa

${ }^{6}$ Según datos proporcionados por la Oficina del INEM de San Juan de Aznalfarache, con fecha 13 de febrero de 2001 . 
que las mujeres demandan empleo a edades más tempranas que los hombres, evidenciándose este fenómeno más claramente en el periodo de los 25 a los 35 años.

- Del total de personas demandantes activos de empleo, la gran mayoría poseen E.G.B. (947 personas), o simplemente Certificado de Escolaridad (819 personas). A estos le siguen quienes han realizado B.U.P. (240 personas) y Formación Profesional (178 personas). Tan sólo 91 personas poseen Título de Graduado Superior y 66 personas el Título de Grado Medio. Sin estudios aparecen reflejadas 24 personas, aspectos todos con los que pudimos comprobar que el nivel de estudios de la población de San Juan era relativamente bajo. En el gráfico que presentamos a continuación (Gráfico 1) exponemos más claramente estos datos, pues los consideramos de interés desde el estudio realizado. En él aparece representado de forma gradual, es decir, de menor a mayor el nivel formativo / educativo de la población de San Juan.

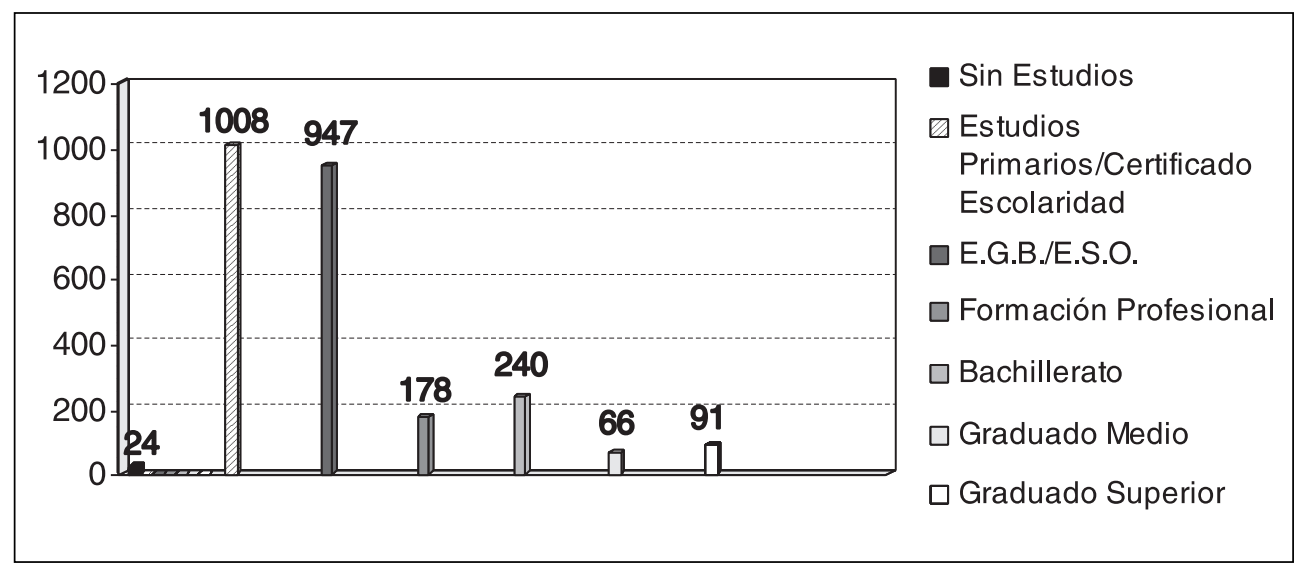

GRÁFICO 1.

Población de San Juan por niveles de instrucción.

- Así, se observa en la zona la existencia de barriadas con necesidades de transformación social, concretamente la barriada Santa Isabel, donde existen muchas necesidades a cubrir especialmente en determinados colectivos. Uno de ellos es el de las mujeres jóvenes, que se caracterizan por ser una población significativa, con escasa formación y una alta demanda de empleo.

Estas necesidades que extraemos del contexto guardan estrecha conexión con los datos obtenidos en las entrevistas realizadas a las diferentes profesionales que trabajaban en nuestro ámbito de intervención. Una de las dimensiones extraídas de este análisis fue la relativa al contexto-barrio, cuyas categorías hacen referencia al bajo nivel socio-económico-cultural de la barriada, a la situación de discriminación socio-laboral que sufren las mujeres en la misma debido a patrones culturales (etnia gitana), a problemas de convivencia asociados a esta problemática, al valor que la familias otorgan a la educación de sus hijos, al papel que tiene la escuela en este contexto, etc. De todo ello se deduce, a primera vista, la necesidad de poner en marcha actuaciones de mejora que den respuesta a dichas situaciones. 


\section{Necesidades expresadas y sentidas por parte de las destinatarias del programa}

Los resultados que a continuación se exponen son los que se obtienen del análisis del cuestionario y de la escala ESCE, los cuales dan respuesta al primero de los objetivos planteados. Entre los principales resultados, y centrándonos en la situación actual que viven estas mujeres, destacamos que el $100 \%$ de las mismas no está cursando ningún estudio, ya que abandonan la formación a edades tempranas. Dicho abandono es debido a diferentes motivos, los cuales presentamos en el gráfico 2 .

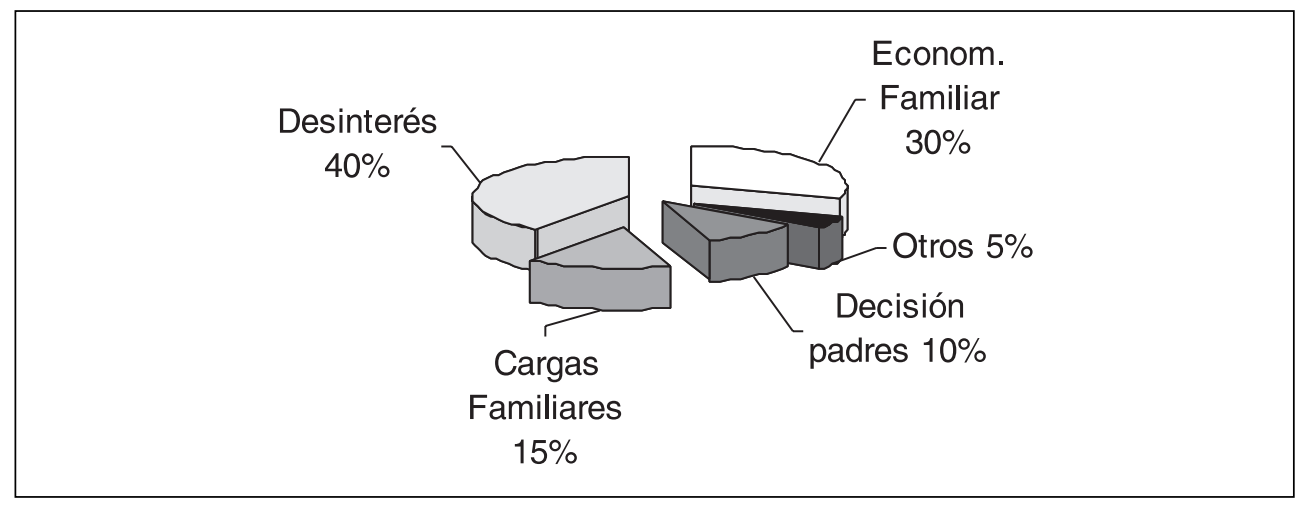

GRÁFICO 2.

Motivo de abandono de la educación / formación.

Las chicas manifiestan como causas principales de abandono la situación económica que tienen sus familias (30\%) y el desinterés de ellas mismas ante la formación / educación (40\%). También nos encontramos con un porcentaje relevante de las chicas, un $15 \%$, que abandonan los estudios por la dedicación a cuidados familiares u otras ocupaciones, como son las tareas del hogar, la ayuda al trabajo familiar, o la realización de otros trabajos debido a la necesidad económica, y que se sitúan en sectores sumergidos. Con menores porcentajes, aunque llamativos, aparece el abandono de la formación por decisión paterna (un $10 \%$ ). En este sentido, algunas de las chicas manifiesta haber abandonado el sistema educativo por deseo expreso de sus padres.

La decisión personal juega un papel relevante y es un hecho que, en nuestra opinión, debemos unirlo con el factor de motivación dentro del sistema educativo, con el fracaso escolar, así como con las cuestiones étnicas y culturales. Esto que aportamos cobra sentido si tenemos presente que algunas de las chicas son gitanas $(\mathrm{fr}=5)$. Nos planteamos al respecto un interrogante: ¿responde la escuela a los intereses de estas chicas?, el desinterés y el fracaso escolar que este grupo expresa ¿es intrínseco de ellas, o más bien del sistema educativo que no da respuesta a sus necesidades? ... De aquí resaltamos el importante papel que juega la situación familiar en los procesos de desarrollo vital / profesional y en la formación de estas mujeres para el mismo.

De esta manera, nos encontramos con que el nivel formativo de estas chicas es muy bajo, algo que hacemos visible en el gráfico 3. Muchas de ellas no poseen estudios, un $45 \%$, y de las que tienen mayor nivel de formación, un 55\%, cursan hasta E.G.B. o E.S.O., 
aspecto que les dificulta mucho el acceso al mercado laboral. De esta manera, el $90 \%$ de la muestra se encuentra en situación de desempleo (Gráfico 4), y únicamente el 10\% trabaja, aunque lo hace en unas determinadas condiciones (trabajos sumergidos).

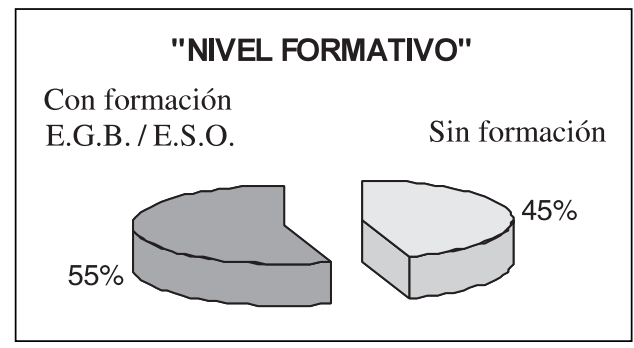

GRÁFICO 3.

Nivel formativo actual que poseen las chicas.

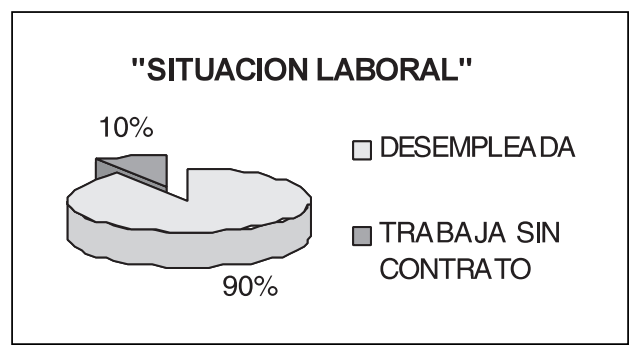

GRÁFICO 4.

Situación laboral actual de las chicas.

El "tipo de trabajo" al que acceden (Gráfico 5) es al cuidado de personas y /o a tareas domésticas, encontrando también trabajo -temporal y sin contrato- en supermercados. No obstante, a pesar del porcentaje elevado de abandono escolar, el $90 \%$ de estas mujeres da mucho valor a la formación a la hora de acceder a un puesto de trabajo. Un $80 \%$ de las mismas parece ser consciente de sus carencias formativas para insertarse socio-laboralmente en su entorno. Un $20 \%$ alude a sus situaciones familiares como obstáculo y /o limitación principal para ello (Gráfico 6).

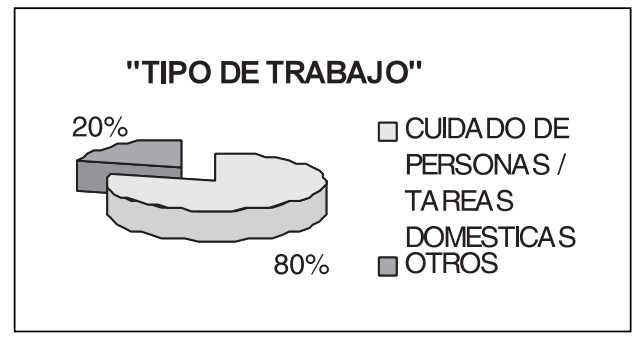

GRÁFICO 5.

Tipo de trabajo al que acceden las chicas.

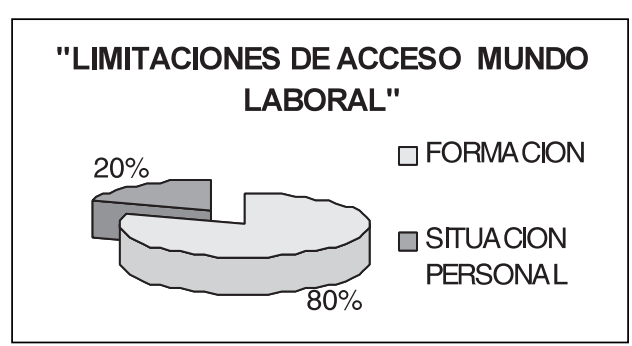

GRÁFICO 6. Limitaciones para la inserción socio-laboral de las chicas.

De esta manera quedan patentes las relaciones que se establecen entre el acceso al empleo de estas chicas y sus situaciones familiares. Éstas fluyen bien sea considerando el tiempo que le quitan los cargos u ocupaciones familiares, como por el hecho de que están inmersas en un contexto socio-familiar donde se percibe el trabajo de la mujer desde un punto de vista más reproductivo que productivo. La situación es percibida desde el conformismo, la cotidianidad y la estabilidad (dificultad de cambio). 
Con respecto a las variables consideradas en la escala ESCE se tienen en cuenta las puntuaciones totales de la misma. Éstas son interpretadas teniendo como referente el perfil de la escala que su autora propone para ello (Rodríguez Moreno, 1999). Esto nos permite conocer si una determinada puntuación es alta, media o baja en relación a las escalas y subescalas que comprende el instrumento. En la siguiente tabla (tabla 1) presentamos las medianas alcanzadas en las distintas sub-escalas del mismo, las cuales pasamos a comentar.

TABLA 1: Resultados obtenidos de la aplicación de la escala ESCE.

\begin{tabular}{|l|c|c|}
\hline \multicolumn{1}{|c|}{ SUBESCALAS } & MEDIANAS & $\begin{array}{c}\text { CALIFICACIÓN SEGÚN } \\
\text { PERFIL DE LA ESCALA }\end{array}$ \\
\hline Cantidad de información & 6,5 & Poca \\
\hline Exploración sistemática intencional & 6 & Poca \\
\hline Exploración del medio & 17,5 & Escasa \\
\hline Exploración de sí mismo & 17,5 & Aceptable \\
\hline Ansiedad & 1 & Poca \\
\hline Satisfacción con la información & 8,5 & Poca \\
\hline Certeza en obtención de información & 10,5 & Razonable \\
\hline Instrumentalidad externa & 22 & Buena \\
\hline Instrumentalidad interna & 20 & Buena \\
\hline Importancia puesto de trabajo preferido & 17 & Mucha \\
\hline Expectativas reales de empleo & 9 & Alguna \\
\hline Estrés exploratorio & 11,5 & Bastante \\
\hline Estrés decisorio & 11 & Bastante \\
\hline
\end{tabular}

En lo que se refiere a la primera sub-escala, podemos considerar que las mujeres están muy poco informadas en lo relativo al mundo laboral. Las actividades que realizan para preparar su futuro profesional, ya sean prácticas o trabajos para explorar una determinada profesión, búsqueda de medios de formación, u otras, son muy poco sistemáticas, no teniendo conciencia de ello. De esta manera, perciben su acceso al mercado laboral no como un proceso, sino como una actividad puntual y concreta a corto plazo. Unido a este factor, las chicas poseen una escasa exploración del medio, lo que se traduce en escasa iniciativa y motivación personales en momentos claves del proceso de búsqueda de empleo (búsqueda de información y/o acceso a servicios de orientación y consulta). Por tanto, tales recursos son desconocidos para las chicas y no son tenidos en cuenta a la hora de buscar trabajo en su entorno más inmediato.

Respecto a la sub-escala cuatro, exploración de sí mismo, se considera aceptable la reflexión que estas mujeres hacen sobre sí mismas. Es decir, el hecho de que se planteen muy a menudo la relación entre el pasado escolar y el futuro profesional, junto a las repercusiones que dicha conexión (educación-trabajo) puede tener para ellas. No obstante, estas chi- 
cas manifiestan muy poca ansiedad ante la preparación de su futuro profesional, lo que se traduce en pasividad e inactividad ante el mismo. Se muestran muy poco satisfechas con la información de que disponen en el momento sobre trabajos y áreas ocupacionales de interés, aunque parecen estar "razonablemente" seguras de sus preferencias profesionales.

En cuanto a la instrumentalidad, tanto externa como interna, el grupo refleja poseer una buena planificación y una aceptable habilidad para autoevaluarse y aprender sobre sí mismas. Se observa además, la importancia que tiene para ellas el acceder a profesiones de interés, aunque las expectativas reales que muestran hacia su empleabilidad no son demasiado altas. Este hecho nos refleja la desmotivación que este colectivo posee de cara a su inserción sociolaboral. En las dos últimas sub-escalas, observamos que estas mujeres muestran bastante estrés, tanto en momentos en los que tienen que enfrentarse a situaciones de búsqueda de información o trabajo y /o entrevista de selección profesional (estrés exploratorio), como en situaciones en las que tienen que tomar una decisión importante en cuanto a su presente-futuro profesional (estrés decisorio).

\section{Necesidades expresadas por las profesionales con respecto al desarrollo profesional de las chicas}

En este apartado exponemos los resultados principales procedentes del análisis cualitativo en torno a las "Necesidades de Orientación y Formación" que, según las diferentes profesionales entrevistadas, poseen las destinatarias de nuestra intervención. Nos centramos en dicha información aunque es necesario referir que esta interactúa con la procedente de las otras dimensiones de análisis, como el contexto social donde se desarrolla el programa y las necesidades sentidas y manifestadas por el grupo diana. En la tabla siguiente (tabla 2) presentamos las categorías que emergen de este análisis y que dan respuesta al segundo de los objetivos planteados en este trabajo.

\section{TABLA 2: Sistema de categorías relativo a la dimensión "Necesidades de Orientación y Formación".}

\begin{tabular}{|c|c|c|}
\hline DIMENSIÓN & CATEGORÍA & CÓDIGO \\
\hline & - Cambio de Mentalidad & MEN \\
& - Orientación Laboral & OLA \\
Necesidadesde & - Exploración del entorno & OLA a \\
$\begin{array}{c}\text { Orientacióny } \\
\text { Formación }\end{array}$ & - Autoestima / auto-conocimiento & OLA b \\
& - Técnicas de búsqueda empleo & OLA c \\
& - Toma de decisiones y resolución de problemas & OLA d \\
& - Habilidades sociales & HAS \\
& Motivación / aspiraciones destinatarias & ASP \\
\hline
\end{tabular}

La categoría cambio de mentalidad hace referencia a la forma de pensar y a las actitudes de las personas que viven en esta zona (mujeres destinatarias del programa, sus familias, y demás personas de la barriada). En este sentido, nos aparece reflejada la necesidad en el contexto-barrio de un cambio - por parte de todas las personas que viven en él- en las maneras y en las formas de relaciones y de convivencia. Se alude a cambios en actitudes con 
respecto a la vida y al concepto que se tiene de la misma, a las concepciones sobre la educación, así como a la importancia que las familias le otorgan a la formación y al acceso al empleo de sus hijas. Todos estos aspectos guardan relación con el propio contexto social donde se inserta la barriada, el nivel sociocultural y económico de la misma, así como con la función que desempeña la escuela en él.

La segunda de las categorías, denominada motivación /aspiraciones de las destinatarias, alude a la motivación que tienen las mujeres hacia el aprendizaje, la formación, la búsqueda de empleo y, en general, hacia la vida (desarrollo vital / profesional). Respecto a ella podemos decir que las aspiraciones que estas chicas poseen no son nada "exigentes". El acceso al empleo se considera una necesidad prioritaria debido a la precaria situación económica que viven sus familias. En este sentido, su principal interés es el acceso al empleo para ganar el dinero que necesitan para subsistir. Sin cualificación básica y adoptando el cauce más rápido que tienen para su colocación, las chicas acceden a trabajos sumergidos y precarios.

"Su principal motivación es ganar dinero pronto, entonces dicen... dejo de estudiar, además creen que van a encontrar trabajo pronto, aunque sea limpiando casas... entonces dejan los estudios, como en casa les hace falta ganar dinero, pues entonces la familia tampoco les pone demasiado impedimento ¿no?, entonces, claro, después es muy difícil, porque hasta los 18 años no pueden entrar en el Centro de Adultos..." (Entrevista A, líneas 42-49).

Con la categoría de Orientación laboral hacemos referencia a cuestiones relacionadas con el proceso de búsqueda de empleo y el acceso al mundo del trabajo. Concretamente, dentro del plano laboral detectamos necesidades en cuanto al proceso general de búsqueda de empleo. Éstas se hacen patente tanto en lo relativo a sus fases como a las actividades concretas a realizar en el mismo. A todo ello se une el concepto propio de búsqueda de empleo que tienen las chicas como una actividad puntual que se realiza en un momento concreto de sus vidas. Este aspecto es fundamental para el análisis de la conducta exploratoria.

Otra necesidad importante que se aprecia dentro de esta categoría es la de exploración del entorno. Con ello las profesionales entrevistadas aluden al conocimiento que las chicas poseen del medio en que viven, de los recursos existentes en el mismo y de las posibilidades que les ofrece para acceder al mercado laboral. Estas mujeres tienen muy poco conocimiento del contexto donde viven, y sobre las posibilidades que el entorno les brinda para desarrollarse personal y profesionalmente. Desconocen los recursos que existen en la zona, no saben dónde acudir a la hora de buscar empleo, ni qué hacer para ello.

Dentro de la categoría de Orientación Laboral, las técnicas de búsqueda de empleo se consideran también importantes desde el punto de vista de las profesionales entrevistadas. Con ellas aluden a una serie de técnicas y estrategias, cuyo conocimiento facilitaría el proceso de búsqueda de empleo y la ampliación de posibilidades ocupacionales.

"Porque, por ejemplo, el simple hecho de cómo acercarse a la gente, de cómo hablar, tan simple como una entrevista puede ser de cualquier trabajo, que es decisiva para entrar a trabajar, ese tipo de cosas no la tienen, la disposición, la forma de hablar, de expresarse... la forma de comportarse, cómo sentarse... todo ese tipo de cosas hay que trabajarlas con ellas, porque no saben, no están acostumbradas, no lo han visto nunca...”(Entrevista B, líneas 254-264). 
Con la primera de las sub-categorías se hace referencia a la necesidad de favorecer la autoestima de estas mujeres y de potenciar un mayor y mejor auto-conocimiento, confianza y seguridad en sí mismas, en su valía personal y en sus propias acciones. Con la segunda, se alude a las habilidades sociales y a los procesos comunicativos, a las relaciones con los demás, formas de comportamiento con los otros y ante determinadas situaciones. Tanto una como otra se presentan como necesidades importantes a la hora del desarrollo personal y profesional de estas mujeres, ya que han desarrollado muy poco las habilidades sociales y les cuesta mucho esfuerzo establecer procesos comunicativos y argumentar sus respuestas ante determinadas situaciones. Además, estas chicas poseen muy poco conocimiento sobre sí mismas y tienen muy poca confianza en todo cuanto sienten y hacen. Tales aspectos se consideran limitaciones a superar en sus procesos de búsqueda de empleo.

"tienen poco desarrolladas las habilidades sociales, hay gente que a lo mejor... y mucha gente que ha terminado, que le cuesta mucho trabajo... a lo mejor... para irse a la discoteca sí se van, pero para buscarse la vida tienen pocos recursos, pocas habilidades ¿no?, para trabajar en grupo también tienen pocas habilidades, la autoestima baja, algunas veces por introversión y otras por todo lo contrario, por demasiada extroversión, pero... que son síntomas también de haber desarrollado poco las habilidades sociales y también la autoestima" (Entrevista C, líneas $84-93)$.

"que confíen en ellas, que van a ser capaces, que no se hundan, que es difícil, que no llegas y pegas, que es difícil lo que es el mundo laboral, que no llegas y pegas, pero que bueno, a base de esfuerzo, formación, seguir trabajando e intentándolo puedes ir encauzando tu vida, tu trabajo... a partir de lo que tú aprendas y quieras alcanzar" (Entrevista C, líneas 132-137).

Como se pone de manifiesto por las profesionales entrevistadas, en el terreno laboral es conveniente comenzar por la persona en sí. Esto se debe a que en el grupo diana se detectan grandes carencias en cuanto a la toma de decisiones y a la resolución de problemas. Estas chicas muestran grandes dificultades a la hora de tomar decisiones autónomas y responsables, así como de afrontar y de resolver los problemas que se les presentan en sus vidas de una manera crítica, consciente y activa. Estas chicas no son autónomas y están muy influenciadas en sus pensamientos y acciones por sus familiares, amigos, parejas... Esto entraña, sin lugar a duda, dificultades a la hora de su desarrollo vocacional-profesional.

“en el plano laboral, yo empezaría ante todo por la persona en sí, lo positivo y lo negativo de cada cual, el comportamiento en diferentes situaciones, cómo tomamos las decisiones y resolvemos los problemas que se nos presentan... todo el contenido que, de alguna manera, sirva para fomentar la autonomía y la independencia de las chicas al grupo (...) dependen afectivamente, tanto de sus familias como de los novios, y además... no hacen las cosas por ellas mismas...” (Entrevista C, líneas 177-190, 201-103).

Por tanto, este aspecto de la autonomía e independencia de las chicas, tiene importantes repercusiones a la hora de acceder a puestos de trabajos que están alejados lo más mínimo de la zona en la que este colectivo vive. Un ejemplo claro es la dificultad que éstas manifiestan para desplazarse a pueblos cercanos o a la capital, aún ofreciéndoles la posibilidad de empleo. En este sentido se siguen manteniendo barreras socioculturales y económicas, y se obstaculizan las relaciones interpersonales y la apertura a otros ambientes y /o contextos. Éste pensamos que es un condicionante importante para la inserción sociolaboral de estas mujeres jóvenes. 


\section{Conclusiones de la evaluación de necesidades para el diseño de la intervención orientadora: ejes principales del programa}

A la hora de realizar cualquier intervención es fundamental considerar el contexto de partida y el colectivo con el que trabajamos. El nuestro, en concreto, posee muchas carencias formativas que, unidas a factores ambientales, dificultan aún más su inserción laboral. Observamos cómo las dos constantes en la situación de estas mujeres son el desempleo y la economía sumergida, llegando a desempeñar actividades profesionales que requieren muy poca formación y accediendo a trabajos de baja cualificación. De esta manera, el colectivo femenino en el que nos centramos encuentra serias dificultades para integrarse socio-laboralmente en su contexto. Estas chicas pasan por un doble periodo de transición, que se encuentran íntimamente relacionados. Por un lado, se resalta la transición hacia la vida adulta debido a las características propias de su edad, género y contexto (social y familiar). Por otro, la transición hacia la vida activa debido a la propia situación sociolaboral por la que pasan y con la que se enfrentan.

No obstante, existen determinados factores que agravan y alargan este periodo de transición, de los cuales el desempleo reviste especial importancia. La baja autoestima de estas mujeres, su inmadurez, las dificultades para desarrollar una identidad adulta, la falta de entrenamiento en habilidades sociales y actitudes laborales, el desconocimiento del entorno en el que viven, y un largo etcétera, son factores que condicionan su desarrollo personal y profesional. Es importante considerar el factor referido a la formación, ya que gran parte de las alumnas abandona el sistema educativo a edades tempranas sin poseer la formación mínima ni las herramientas básicas para acceder a la vida adulta y activa.

Por tanto, a la hora de diseñar la intervención orientadora es fundamental partir de las necesidades detectadas, considerando como uno de los objetivos principales el aumento del nivel formativo de estas chicas y su reincorporación en el sistema de formación general, bien fuera dentro del ámbito formal (Centro de Personas Adultas) u otras vías de formación, como por ejemplo la Formación Profesional Ocupacional, que está especialmente diseñada para este colectivo. También se destaca la importancia y la necesidad de la orientación profesional en este campo y contexto, entendida como un proceso que conlleva la búsqueda de alternativas personales-sociales, y el consiguiente desarrollo integral personal-profesional de estas mujeres.

Para ello consideramos necesario ofrecer una serie de técnicas, recursos y estrategias que facilitan la inserción sociolaboral de las chicas. También se hace fundamental fomentar las habilidades sociales, pues ello lleva a un contacto más favorable con el entorno laboral de la zona, y aumenta la motivación y las expectativas vitales / profesionales de las chicas. Con ello aludimos al incremento de posibilidades tanto desde el punto de vista formativo / educativo, como de iniciativa personal, autónoma y crítica con y para su desarrollo vital / profesional. Todos estos aspectos (reincorporación al sistema educativo, búsqueda de alternativas personales-sociales, etc.) son los ejes clave sobre los que ha incidido la intervención orientadora planificada a partir de este análisis de necesidades.

De manera muy breve esbozamos más concretamente los aspectos principales que conforman la intervención orientadora consiguiente al estudio realizado. En primer lugar, la meta última que queremos alcanzar es formular un proyecto de vida (personal y profesional), que promueva la inserción sociolaboral para las mujeres jóvenes de la barriada. Algu- 
nos de los objetivos que formulamos van en la línea de: incrementar la motivación de las chicas, tanto en lo que respecta a la persona, educación / formación, como en lo relativo al trabajo, adquirir una serie de conocimientos, destrezas y actitudes que faciliten su acceso al empleo, ser conscientes y hacer explícitos estereotipos sexuales, intentando mermarlos o reducirlos, facilitar la reincorporación de las chicas que han abandonado la escuela al sistema educativo u otras vías de formación, etc.

Los bloques de contenidos que entendemos relevantes son cinco, los cuales denominamos: Auto-conocimiento / Autoconcepto / Autoestima (bloque 1), Mundo laboral (bloque 2), Técnicas de búsqueda de empleo (bloque 3), Mundo formativo / educativo (bloque 4), Toma de decisiones y elaboración de un proyecto vital / profesional (bloque 5). Por su parte, la metodología seguida se caracteriza por ser activa y participativa, ya que las destinatarias del programa poseen un papel protagonista en él. Deben llevar a cabo un proceso de indagación sobre sí mismas y la realidad que las envuelve, lo que les va a permitir adquirir una serie de destrezas, conocimientos y actitudes favorables para su desarrollo vital / profesional. Pretendemos que, de esta manera, vayan tomando conciencia sobre la responsabilidad que poseen con respecto a sus vidas. También es flexible y abierta, teniendo presente la propia individualidad, así como los cambios que puedan darse durante el desarrollo del programa. Tiene en cuenta los principios del modelo A.D.V.P.: experiencial, heurístico, e integrador (Romero, 1996; 1999).

En cuanto a la estructura organizativa, el programa se desarrolla en diferentes aulas destinadas para ello aunque, en momentos puntuales, se utilizan otros espacios, como el Centro de Personas Adultas, empresas, o el propio barrio. La intervención se diseña para un curso escolar, llevándose a cabo de manera integrada en un proyecto más amplio que se desarrolla con las chicas. Nuestro trabajo se enmarca en el área de formación y capacitación profesional y laboral, siendo la modalidad de intervención grupal directa y de seguimiento individual. Como materiales y recursos para llevar a cabo el programa hemos recurrido a diversas infraestructuras, soportes didácticos y equipamientos, al igual que a diferentes profesionales.

\section{Referencias bibliográficas}

Calvet Mojón, M.; Frau Linares, M. J.; Alcañiz Moscardó, M. (2000). Las mujeres del Braix Maestrat en el siglo XXI: hacia la igualdad en el reparto del trabajo y del tiempo. Castellón. Oficina de Iniciativas Comunitarias del Ayuntamiento de Traiguera.

Carrasco Macías, M. J. (2002). Análisis e incidencias de los estilos directivos de las mujeres en centros escolares de contextos desfavorecidos. Tesis Doctoral. Inédita. Universidad de Huelva.

Carrasco, C. y Rodríguez, A. (1999). "Trabajos y cuidados: hacia una reorganización social del tiempo y del trabajo". Revista de Servicios Sociales y Politica Social, 45, 61-84.

Chait Barnett, R. y Shibley Hyde, J. (2001). "Women, men, work, and family". American Psichology, 56 (10), 781-796.

Cigarini, L. (2000). "El conflicto entre los sexos en el trabajo". DUODA, 19, 13-26.

Donoso, T.; Figuera, P.; Torrado, M. (2000). "Análisis y validación de una escala para medir la conducta exploratoria”. Revista de Investigación Educativa, 18 (1), 201-220.

Donoso, T.; Figuera, P.; Rodríguez, M. L. (1994). "Un instrumento para evaluar la conducta exploratoria en el desarrollo de la carrera profesional". Revista Investigación Educativa, 23, 490-496. 
Gysbers, N. C.; Heppner, M. J.; Johnston, J. A. (2002). Career Counseling. Process, issues, and techniques. United States of America. Chestnut Hill Enterprises, Inc.

Instituto Andaluz de la Mujer (2001). Situación de las mujeres en el mercado de trabajo de Andalucía. Diagnóstico previo. Curso de Experta en Igualdad de Oportunidades. Gabinete de Formación y Empleo. Instituto Andaluz de la Mujer.

Instituto de la Mujer (1999). Mujeres y Sociedad Rural. Entre la inercia y la ruptura. Instituto de la Mujer. Fondo Social Europeo.

Instituto de Estadística de Andalucía (2002). Anuario Andaluz de las Mujeres 2002. Perspectivas de Género. Sevilla. Instituto de Estadística de Andalucía.

Kaufmam Roger, A. (1997). Planificación de sistemas educativos: Ideas Básicas. Concretas. México. Trillas.

Lent, R. W. y Worthington (2000). "On school-to-work transition, career development theories, and cultural validity". The Career Development Quarterly, V48, 4, 376-383.

LIKaDI Formación y Empleo. Instituto Andaluz de la Mujer (2001). Mainstreaming o Enfoque Integrado de Género. Manual de aplicación en proyectos de empleo. Madrid. LIKaDI Formación y Empleo, S.L.

Marí-Klose, M. y Nos Colom, A. (1999). Itinerarios vitales: educación, trabajo y fecundidad de las mujeres. Opiniones y Actitudes $n^{\circ} 27$. Madrid. Centro de Investigaciones Sociológicas (CIS).

Padilla Carmona, M. T. (2001). "Barreras y limitaciones en el desarrollo profesional de la mujer". Portularia, 1, 223-232.

Pérez-Campanero, Ma . P. (1991). Cómo detectar Necesidades de Intervención Educativa. Madrid: Narcea.

Poal Marcet, G. (1993). Entrar, quedarse, avanzar. Aspectos psicosociales de la relación mujer-mundo laboral. Madrid. Siglo XXI.

Radl, R. (2001). "La socialización en función del género". Revista Multidisciplinar de Gerontología, 11 (4), 198-196.

Romero Rodríguez, S. (1996). Aplicaciones del modelo A.D.V.P. al diseño y validación de un programa de orientación para la transición. Universidad de Sevilla. Tesis Doctoral.

Romero Rodríguez, S. (1999). Orientación para la transición de la escuela a la vida activa: una metodología de intervención desde el modelo de Activación del Desarrollo Vocacional y Personal. Barcelona. Laertes.

Rodríguez Moreno, M .L. (1999). Enseñar a explorar el mundo del trabajo. Diagnóstico de las destrezas exploratorias y propuestas de intervención. Málaga. Aljibe.

Rodríguez Moreno, M. L.; Sandín Esteban, M. P.; Buisán Sarradell, C. (2000). "La conducta exploratoria: concepto y aplicaciones en orientación profesional". Revista de Educación, 321, 153-186.

Rodríguez-Piñero, M. y Castellano Burguillo, E. (2003). Políticas de empleo para colectivos desfavorecidos. Curso de Verano. Sede Iberoamericana Santa María de la Rábida. Universidad Internacional de Andalucía. Material Inédito.

Fecha de recepción: 16-09-02

Fecha de revisión: 10-02-04

Fecha de aceptación: 12-05-04 